\title{
Laser Vaporization of Intracoronary Thrombus and Identifying Plaque Morphology in ST-Segment Elevation Myocardial Infarction as Assessed by Optical Coherence Tomography
}

\author{
Yuki Yamanaka, Yoshihisa Shimada 1 , Daisuke Tonomura, Kazunori Terashita, \\ Tatsuya Suzuki, Kentaro Yano, Satoshi Nishiura, Masataka Yoshida, Takao Tsuchida, \\ and Hitoshi Fukumoto
}

Cardiovascular Center, Shiroyama Hospital, 2-8-1 Habikino, Habikino 583-0872, Japan

Correspondence should be addressed to Yoshihisa Shimada; shimada@shiroyama-hsp.or.jp

Received 6 January 2021; Revised 13 June 2021; Accepted 14 July 2021; Published 29 July 2021

Academic Editor: Seif S. El-Jack

Copyright () 2021 Yuki Yamanaka et al. This is an open access article distributed under the Creative Commons Attribution License, which permits unrestricted use, distribution, and reproduction in any medium, provided the original work is properly cited.

\begin{abstract}
Objectives. We evaluated the thrombus-vaporizing effect of excimer laser coronary angioplasty (ELCA) in patients with STsegment elevation myocardial infarction (STEMI) by optical coherence tomography (OCT). Background. Larger intracoronary thrombus elevates the risk of interventional treatment and mortality in patients with STEMI. Methods. A total of 92 patients with STEMI who presented within 24 hours from the onset and underwent ELCA following manual aspiration thrombectomy (MT) were analyzed. Results. The mean baseline thrombolysis in myocardial infarction flow grade was $0.4 \pm 0.6$, which subsequently improved to $2.3 \pm 0.7$ after MT $(p<0.0001)$ and $2.7 \pm 0.5$ after ELCA $(p=0.0001)$. The median residual thrombus volume after MT was $65.7 \mathrm{~mm}^{3}$, which significantly reduced to $47.5 \mathrm{~mm}^{3}$ after ELCA $(p<0.0001)$. Plaque rupture was identified by OCT in only 22 cases $(23.9 \%)$ after MT, but was distinguishable in 36 additional cases after ELCA (total: 58 cases; 63.0\%). Ruptured lesions contained a higher proportion of red thrombus than nonruptured lesions $(75.9 \%$ vs. $43.3 \%, p=0.001)$. Significantly larger thrombus burden after MT $\left(69.6 \mathrm{~mm}^{3}\right.$ vs. $\left.56.3 \mathrm{~mm}^{3}, p<0.05\right)$ and greater thrombus reduction by ELCA $\left(21.2 \mathrm{~mm}^{3} \mathrm{vs} 11.8 \mathrm{~mm}^{3}\right.$, $p<0.01)$ were observed in ruptured lesions than nonruptured lesions. Conclusions. ELCA effectively vaporized intracoronary thrombus in patients with STEMI even after MT. Lesions with plaque rupture contained larger thrombus burden that was frequently characterized by red thrombus and more effectively reduced by ELCA.
\end{abstract}

\section{Introduction}

Intracoronary thrombus burden is a major determinant of adverse clinical outcome in patients with ST-segment elevation myocardial infarction (STEMI). Larger thrombus burden limits the success of percutaneous coronary intervention (PCI) for STEMI, as it increases the rate of procedural complications, such as distal embolization and no reflow phenomenon. It is also associated with worse microvascular dysfunction and greater myocardial damage, thereby significantly affecting mortality $[1,2]$. Therefore, theoretically, reduction of intracoronary thrombus at the culprit lesion improves the outcome of primary PCI and reduces the mortality rate in patients with STEMI.

However, routine thrombus aspiration remains of uncertain value in primary PCI for STEMI. Several randomized studies have demonstrated that manual aspiration thrombectomy (MT) for STEMI prevents the occurrence of the no reflow phenomenon and distal embolization, resulting in better myocardial reperfusion and reduced myocardial infarct size [3-5]. In contrast, larger randomized clinical trials have not shown the clinical benefit of the routine aspiration strategy compared with standard PCI $[2,6]$. These contradictory findings may be attributed to insufficient removal of thrombus using current aspiration thrombectomy devices. 
Several optical coherence tomography (OCT) studies demonstrated that MT had no impact on the reduction of thrombus burden in STEMI, and substantial residual thrombus was observed even after MT at the culprit segment $[7,8]$.

Excimer laser is a unique revascularization device based on the effect of a pulsed ultraviolet (xenon-chloride) laser that directly targets and vaporizes the thrombus. Excimer laser coronary angioplasty (ELCA) has been shown to be safe and effective in acute ischemic-thrombotic coronary syndromes by successful vaporization of intracoronary thrombus at the target lesion [9-12]. Therefore, ELCA may be a useful adjunctive strategy with the potential to further reduce the thrombotic burden which poorly responds to MT. The purpose of this study was to evaluate the efficacy of ELCA following MT in patients with STEMI, by measuring the thrombus burden before and after ELCA using OCT.

\section{Methods}

ELCA for thrombotic coronary lesions has been utilized in our hospital since November 2015. This was a retrospective study of consecutive patients who presented with STEMI within 24 hours after the onset and underwent ELCA under OCT guidance at our hospital. STEMI was defined as continuous chest pain that lasted $>20$ min, electrocardiogram showing new ST-segment elevation $\geq 0.2 \mathrm{mV}$ in at least two contiguous precordial leads or $\geq 0.1 \mathrm{mV}$ in at least two contiguous limb leads, and angiographic identification of a coronary thrombotic stenosis or occlusion. This study was approved by the ethics committee of our hospital and conducted in accordance with the principles of the Declaration of Helsinki. Written informed consent was provided by all patients.

Heparin was administered intravenously with an initial bolus of $5,000 \mathrm{U}$, followed by periodic shots to maintain an activated clotting time between 200 and $300 \mathrm{~s}$ during the procedure. Dextran of mean molecular weight $40 \mathrm{kDa}$ (50-100 mL of $10 \%$ dextran-40 per hour) was also used during the procedure to prevent thromboembolic complications. After initial coronary angiography, MT was first performed using a manual thrombectomy device (Thrombuster III GR; Kaneka Corp., Osaka, Japan or Export Advance; Medtronic, Dublin, Ireland) and repeated at least thrice until there was no visible thrombotic material in the aspirate. ELCA was subsequently performed using a pulsed xenon-chloride excimer laser system with a wavelength of $308 \mathrm{~nm}$; pulse duration of $135 \mathrm{~ns}$; and output of $175 \mathrm{~mJ} /$ pulse (CVX-300P; Royal Philips, Amsterdam, Netherlands). The laser atherectomy catheters $\left(\mathrm{ELCA}^{\mathrm{TM}}\right.$ Coronary Laser Atherectomy Catheter; Royal Philips) were available in sizes of $0.9,1.4,1.7$, and $2.0 \mathrm{~mm}$, and the default energy parameters were set at a fluence of $45 \mathrm{~mJ} / \mathrm{mm}^{2}$ and a repetition rate of $25 \mathrm{~Hz}$. The size of the catheter, maximum fluency, and repetition rate (up to $60 \mathrm{~mJ} / \mathrm{mm}^{2}$ and $40 \mathrm{~Hz}$, respectively) and the number of passes were at the operator's discretion. The laser vaporizing manner employed in this study was previously described [9]. The procedure was followed by balloon dilatation and finalized with drug-eluting stent implantation or drug-coated balloon utilization. All patients received loading doses of oral antiplatelet agents immediately after the procedure, consisting of aspirin $(200 \mathrm{mg})$ and P2Y12 inhibitor (clopidogrel $300 \mathrm{mg}$ or prasugrel $20 \mathrm{mg}$ ) if they were not pretreated with a dual antiplatelet therapy.

A frequency-domain OCT imaging catheter system (Dragonfly OPTIS/ILUMIEN OPTIS OCT Imaging System; Abbott Vascular Inc., Santa Clara, CA, USA) was used in this study. The OCT imaging acquisition was performed after MT, ELCA, and final ballooning, with a pullback speed of $18 \mathrm{~mm} / \mathrm{s}$ and acquisition of 180 frames/s. Intracoronary administration of isosorbide dinitrate $(200-500 \mathrm{mg})$ was performed prior to each pullback. The acquired OCT raw data were stored and exported in digital format for offline analyses using a dedicated offline review system with a semiautomated contour-detection software (Abbott Vascular Inc). All OCT images were analyzed by two experienced investigators (Y.Y. and D.T.). In case of any discordance between the observers, a consensus reading was obtained from a third investigator (Y.S.).

The entire length of the atherothrombotic lesion plus $5 \mathrm{~mm}$ proximal and distal reference segments were included in the analysis. Intracoronary thrombus was defined as an irregular mass attached to the luminal surface or floated from the vessel wall and characterized according to the signal characteristics $[1,13]$. The predominant type of thrombus was categorized according to the signal characteristics: highly backscattering with high attenuation (red thrombus), less backscattering and homogeneous with low attenuation (white thrombus), or a mixture of both (mixed thrombus) $[13,14]$. Thrombus and lumen area were measured by planimetry at $1 \mathrm{~mm}$ intervals with the automatic lumen contour and supplementary manual correction, and the thrombus and lumen volume were calculated by multiplying the areas in each frame by the number of frames $[1,13,15]$. The distance between the most distal and proximal frames that showed intraluminal material suggestive of thrombus defined the thrombus length [13].

The culprit lesion was categorized into four groups: plaque rupture, plaque erosion, calcified nodule, and unidentified $[1,14]$. Plaque rupture was defined by the presence of fibrous-cap discontinuity with a cavity in the plaque. Plaque erosion was defined by the presence of an attached thrombus overlying an intact and visualized plaque or irregular luminal surface with no evidence of fibrous-cap disruption. Calcified nodule was defined by the presence of nodular protruding calcium or luminal surface disruption over a calcified plaque associated with the thrombus. The culprit lesions, unable to be recognized based on the aforementioned criteria by the three observers, were classified as unidentified.

Normally distributed continuous variables are expressed as mean \pm standard deviation, while nonnormally distributed continuous variables are presented as median and interquartile range. Categorical variables are expressed as counts and percentages. Repeated-measures analysis of variance with post hoc Tukey's honest significant difference test was applied to compare serial parameters after each 
procedure. The Mann-Whitney $U$ test or Pearson's chisquared test was applied to examine differences between the lesions with ruptured plaque and those with nonruptured plaque. A $p$ value $<0.05$ was considered statistically significant. All statistical analyses were performed using the JMP statistical software (version 13.2; SAS Institute, Cary, NC, USA).

\section{Results}

From December 2015 to December 2019, 352 consecutive patients with STEMI who presented within $24 \mathrm{~h}$ after the onset of symptoms underwent emergent PCI at our hospital. OCT was not attempted under the following conditions: patients with cardiogenic shock $(n=47)$; STEMI caused by in-stent thrombosis $(n=21)$ or spontaneous coronary artery dissection $(n=4)$; impaired kidney function (serum creatinine $\geq 1.5 \mathrm{mg} / \mathrm{dl} ; n=82)$; left main disease $(n=16)$; smallvessel disease $(<2 \mathrm{~mm}$ in diameter; $n=38)$; extremely tortuous vessels $(n=8)$; heavily calcified vessels $(n=23)$; or other conditions the physicians considered inappropriate $(n=7)$. Therefore, 106 patients underwent OCT-guided PCI with the intention to perform MT followed by ELCA (Figure 1). Patients in whom it was not possible to advance the manual aspiration catheter $(n=1)$ or OCT catheter $(n=2)$ distal to the culprit lesion before ELCA or those with poor OCT image quality $(n=11)$ were excluded from this study. Finally, a total of 92 lesions in 92 patients with STEMI who underwent aspiration thrombectomy followed by ELCA of the infarct-related artery were analyzed in this study.

Baseline patient, lesion, and procedural characteristics are shown in Tables 1 and 2. A laser size of $1.7 \mathrm{~mm}$ was used in $>80 \%$ of cases, mainly because it is the maximum laser size compatible with a $6 \mathrm{Fr}$ guide catheter. The excimer laser catheter was successfully crossed distal to the culprit lesion in all cases without any balloon predilatation. The mean baseline thrombolysis in myocardial infarction (TIMI) flow grade on initial angiography was $0.4 \pm 0.6$. The TIMI flow grade was significantly improved by MT to $2.3 \pm 0.7$ $(p<0.0001)$ compared to that at baseline and subsequently improved by ELCA to $2.7 \pm 0.5(p=0.0001)$ compared to that at post MT (Figure 2). None of the cases developed ELCA-induced perforation, angiographical distal embolization, or flow-limiting dissection. ELCA-induced TIMI flow deterioration was observed in two cases $(2.2 \%)$; however, no visible distal embolization was detected. TIMI 3 flow was achieved in 87 of 92 patients (94.6\%) after adjunctive balloon angioplasty and 90 of 92 patients (97.8\%) after final devices (drug-eluting stent or drug-coated balloon).

The results of the OCT analysis are shown in Table 3. Compared with those after MT, ELCA significantly reduced thrombus volume (from $62.8 \mathrm{~mm}^{3}$ to $46.2 \mathrm{~mm}^{3}, p<0.0001$ ), resulting in larger lumen volume (from $70.0 \mathrm{~mm}^{3}$ to $87.7 \mathrm{~mm}^{3}, p<0.0001$ ) and minimum lumen area (from $1.4 \pm 0.7 \mathrm{~mm}^{2}$ to $2.4 \pm 0.9 \mathrm{~mm}^{2}, p<0.0001$ ) (after MT and after ELCA, respectively).

Using the OCT images after MT, plaque rupture at the culprit lesion was identified in only 22 of 92 cases (23.9\%), but was successfully distinguished in another 36 cases (total 58 cases: 63.0\%) after ELCA (Figures 3 and 4). Among the other cases, OCT after ELCA demonstrated the morphology of plaque erosion in 23 cases (25.0\%) (Figure 5) and calcified nodule in seven cases (7.6\%) (Figure 6), whereas the remaining four cases $(4.3 \%)$ were unidentifiable. Red thrombus was observed more frequently in lesions with ruptured plaque than those with nonruptured plaque $(75.9 \%$ vs. $43.3 \%$, respectively, $p=0.001$ ) (Table 4 ). Although the residual thrombus burden after MT was larger in lesions with ruptured plaque $\left(69.6 \mathrm{~mm}^{3}\right.$ vs. $56.3 \mathrm{~mm}^{3}$, respectively, $p<0.05)$, the vaporizing effect by ELCA on reducing the thrombus burden was more pronounced in lesions with ruptured plaque than those with nonruptured plaque $\left(21.2 \mathrm{~mm}^{3}\right.$ vs. $11.8 \mathrm{~mm}^{3}$, respectively, $\left.p<0.01\right)$ (Figure 7).

\section{Discussion}

Thrombus burden at the culprit lesion remains an important risk factor for cardiac damage and mortality following primary PCI in patients with STEMI $[1,2]$. Mechanical removal of the occlusive thrombus appears to be a logical option for the treatment of these highly thrombotic lesions, and several clinical trials demonstrated that MT during primary PCI resulted in a lower risk of distal embolization and no reflow phenomenon, better ST-segment resolution, preserved microvascular integrity, lower myocardial damage, and reduced mortality rate [3-5]. However, recent large randomized trials and a historical cohort study failed to show an additional benefit of routine thrombus aspiration in patients with STEMI $[2,6]$. This may be attributed to insufficient removal of the thrombus at the culprit lesion by the currently available MT devices. Previous OCT studies demonstrated that MT did not significantly reduce the thrombus burden at the culprit lesions versus lesions without MT $[7,8]$. In our study, we also observed a substantial amount of OCT-identified residual thrombus even after repeated MT.

Our study demonstrated that ELCA additionally reduced thrombus burden at the culprit lesion and restored luminal flow space, as measured using OCT even after MT. In addition, ELCA significantly enhanced the restoration of anterograde TIMI flow of the infarct-related artery without distal embolism. Previous reports have shown ELCA to be feasible and efficient in primary PCI for STEMI, demonstrating improvement in TIMI flow, TIMI frame count, and TIMI myocardial blush grade [9-12]. The ELCA effect is based on three unique mechanisms of a pulsed xenonchloride laser (photochemical, photothermal, and photomechanical), and its thrombus-vaporizing effect may exceed the simple mechanical effect of a manual aspiration catheter on reducing the thrombus burden [16].

The products of in vitro excimer laser thrombolysis are reported to be $<10$ micron in size [17], and previous clinical studies reported that ELCA achieved a higher rate of tissue-level reperfusion (myocardial blush grade, ST-segment resolution) than MT and balloon angioplasty in patients with STEMI $[18,19]$. However, even invisible microdebris may cause microcirculatory impairment during ELCA, such as during 


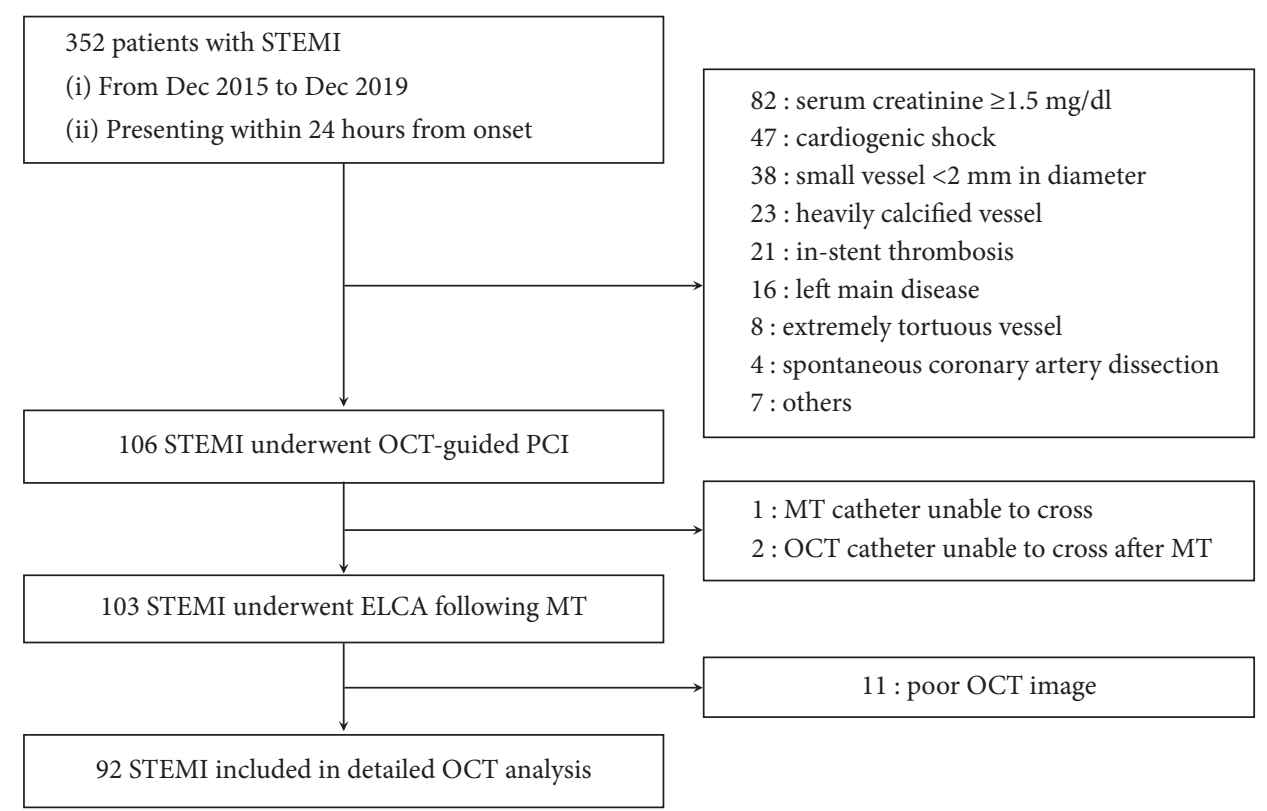

FIgURE 1: Study flow chart. ELCA, excimer laser coronary angioplasty; MT, manual aspiration thrombectomy; OCT, optical coherence tomography; and STEMI, ST-segment elevation myocardial infarction.

TABle 1: Patient characteristics.

\begin{tabular}{lc}
\hline Variable & Value \\
\hline Number of patients & 92 \\
Age (years) & $63.5 \pm 12.6$ \\
Male sex & $77(83.7 \%)$ \\
Hypertension & $59(64.1 \%)$ \\
Diabetes mellitus & $38(41.3 \%)$ \\
Hemoglobin Alc (\%) & $6.5 \pm 1.4$ \\
Dyslipidemia & $54(58.7 \%)$ \\
LDL-cholesterol (mg/dL) & $126.4 \pm 38.8$ \\
Current smoking & $45(48.9 \%)$ \\
Serum creatinine (mg/dL) & $0.85 \pm 0.78$ \\
Prior MI & $1(1.1 \%)$ \\
Prior PCI & $1(1.1 \%)$ \\
Symptom onset to hospital arrival (h) & $5.5 \pm 5.5$ \\
Baseline CPK (IU/L) & $460 \pm 762$ \\
Baseline CPK-MB (IU/L) & $43 \pm 65$ \\
Peak CPK (IU/L) & $2,868 \pm 2,391$ \\
Peak CPK-MB (IU/L) & $272 \pm 239$ \\
\hline
\end{tabular}

Data are presented as $n(\%)$ or mean \pm standard deviation. $\mathrm{CPK}=$ creatine phosphokinase; $\mathrm{CPK}-\mathrm{MB}=$ creatine phosphokinase myocardial band; $\mathrm{LDL}=$ low-density $\quad$ lipoprotein; $\quad \mathrm{MI}=$ myocardial $\quad$ infarction; $\mathrm{PCI}=$ percutaneous coronary intervention.

rotational coronary atherectomy, resulting in slow or no reflow phenomenon. In addition, microcirculatory impairment can occur more frequently in the setting of acute myocardial infarction because of reperfusion injury, platelet aggregation, vasospasm, or tissue edema. In this study, ELCA-induced TIMI flow deterioration was observed in two cases $(2.2 \%)$. We assume that this might be attributable to the microcirculatory disorder, albeit no visible distal embolization was detected.

More thorough removal of thrombus by ELCA may be beneficial, particularly to patients with large thrombus burden. In our study, the effect of ELCA on thrombus dissolution was more pronounced in lesions with extensive thrombus. This finding is in line with previous studies which showed significantly higher acute laser gain in lesions with larger thrombus burden $[9,12]$. However, estimating thrombus burden by angiography alone is often difficult, and a previous study reported that the angiographic thrombus grade does not correlate with intracoronary thrombus volume as measured by OCT [20]. OCT is currently the most reliable method of thrombus quantification in vivo $[13,15]$. Therefore, it may potentially stratify patients with high thrombus burden who further benefit from thrombus dissolution therapy, such as ELCA.

Vaporizing thrombus by ELCA may assist in understanding the pathophysiology of the culprit lesion in STEMI. In this study, a significant amount of residual thrombus hampered the assessment of the underlying plaque morphology in most cases even after MT. This was mainly because of the fundamental limitation of the OCT signal being unable to penetrate red cell-rich thrombus. Even with its high resolution of OCT imaging, the majority of plaque ruptures were identifiable after thrombus vaporization by ELCA. Previous studies have reported that the exposed necrotic core of ruptured plaque is highly thrombogenic and contains larger amounts of red thrombus compared with other plaque phenotypes [21, 22]. Our study demonstrated that adequate removal of sizable red thrombus, which causes rapid attenuation of the OCT signal, may be required for more precise detection of ruptured plaque in the culprit lesion in STEMI.

Aggressive thrombus removal and understanding the morphology of the culprit lesion may potentially lead to better management of the therapeutic strategies. In STEMI, the pathological culprit lesion is often located proximal or distal to the angiographical occlusion site [23]; therefore, 
TABLE 2: Lesion and procedural characteristics.

\begin{tabular}{|c|c|c|}
\hline Variable & & Value \\
\hline \multicolumn{3}{|c|}{ Culprit lesion location } \\
\hline $\mathrm{LAD}$ & & $41(44.6 \%)$ \\
\hline $\mathrm{LCx}$ & & $11(12.0 \%)$ \\
\hline RCA & & $40(43.5 \%)$ \\
\hline \multicolumn{3}{|c|}{ Angiographic parameters } \\
\hline Reference diameter (mm) & & $2.98 \pm 0.51$ \\
\hline Lesion length $(\mathrm{mm})$ & & $19.0 \pm 4.0$ \\
\hline Minimal lumen diameter (mm) & & $0.15 \pm 0.26$ \\
\hline Diameter stenosis $(\%)$ & & $94.6 \pm 9.1$ \\
\hline \multicolumn{3}{|c|}{ Initial TIMI flow grade } \\
\hline TIMI 0 & & $64(69.6 \%)$ \\
\hline TIMI 1 & & $23(25.0 \%)$ \\
\hline TIMI 2 & & $5(5.4 \%)$ \\
\hline \multicolumn{3}{|c|}{ Access site and the size of guide catheter } \\
\hline Transradial (6 Fr) & & $84(91.3 \%)$ \\
\hline Transfemoral (6 Fr/8 Fr) & & $7 / 1(7.6 \% / 1.1 \%)$ \\
\hline \multicolumn{3}{|c|}{ Laser catheter used } \\
\hline $1.4 \mathrm{c}$ & & $15(16.3 \%)$ \\
\hline $1.7 \mathrm{c}$ & & $74(80.4 \%)$ \\
\hline $1.7 \mathrm{e}$ & & $2(2.2 \%)$ \\
\hline $2.0 \mathrm{c}$ & & $1(1.1 \%)$ \\
\hline Maximum energy fluency $(\mathrm{J})$ & & $56.9 \pm 6.2$ \\
\hline Maximum repetition rate $(\mathrm{Hz})$ & & $27.7 \pm 5.7$ \\
\hline Laser pulses delivered & & $2,338 \pm 1,380$ \\
\hline \multicolumn{3}{|c|}{ Final device } \\
\hline DCB & & $87(94.6 \%)$ \\
\hline Size of DCB (mm) & & $3.28 \pm 0.46$ \\
\hline Total length of DCB (mm) & & $28.8 \pm 12.0$ \\
\hline DES & & $5(5.4 \%)$ \\
\hline Size of DES (mm) & & $3.35 \pm 0.22$ \\
\hline Total length of DES (mm) & & $34.8 \pm 12.1$ \\
\hline
\end{tabular}

$\mathrm{DCB}$ = drug-coated balloon; $\mathrm{DES}=$ drug-eluting stent; $\mathrm{LAD}=$ left anterior descending artery; $\mathrm{LC}=$ left circumferential artery; $\mathrm{RCA}=$ right coronary artery; TIMI $=$ thrombolysis in myocardial infarction .

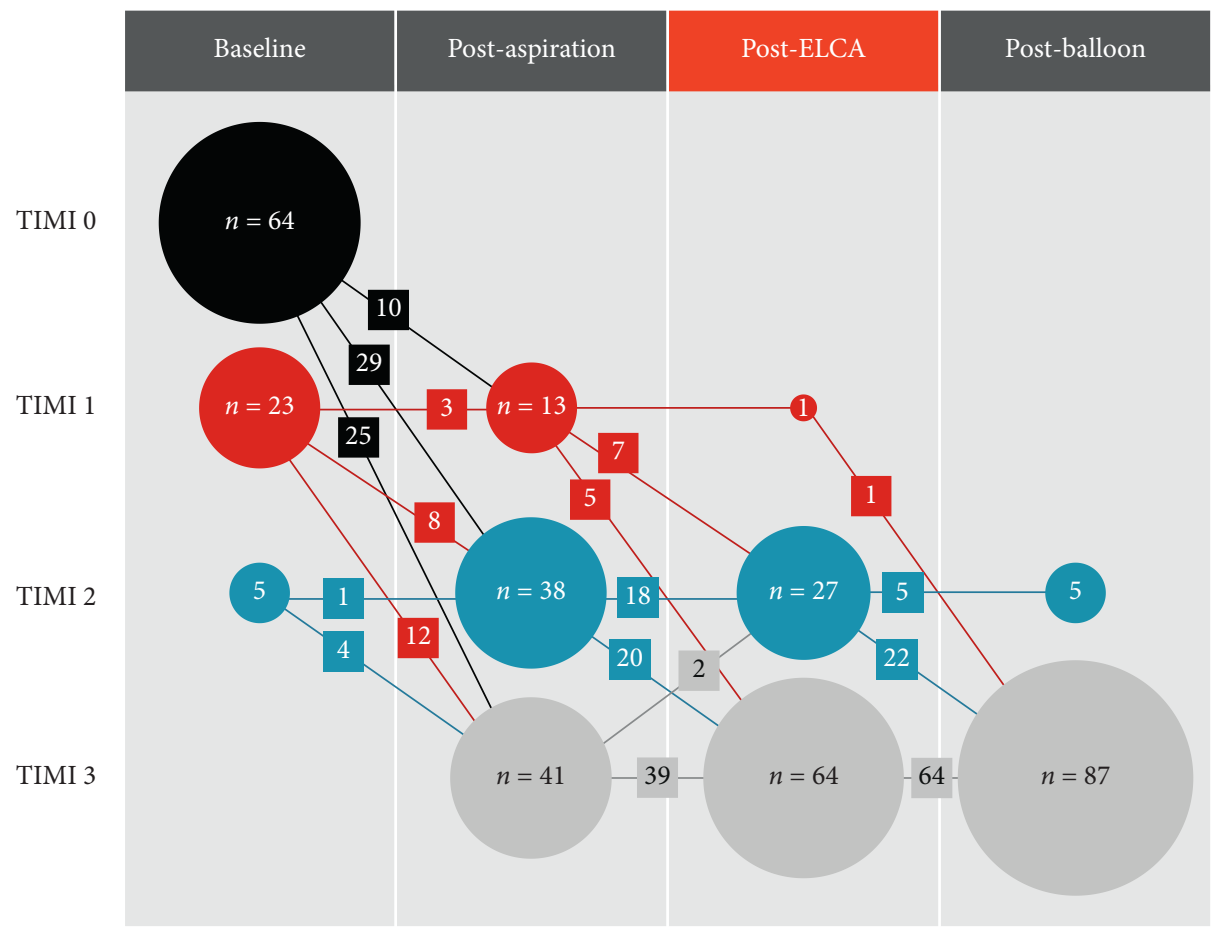

Figure 2: TIMI flow grade and the number of the patients after each of the procedures. TIMI, thrombolysis in myocardial infarction. 
TABLE 3: Results of optical coherence tomography results.

\begin{tabular}{|c|c|c|}
\hline Variable & & Value \\
\hline \multicolumn{3}{|c|}{ Thrombus volume $\left(\mathrm{mm}^{3}\right)$} \\
\hline After manual thrombectomy & & $62.8(41.4-85.4)$ \\
\hline After ELCA & & $46.2(28.3-62.3)$ \\
\hline After ballooning & & $13.3(4.0-27.7)$ \\
\hline \multicolumn{3}{|c|}{ Luminal volume $\left(\mathrm{mm}^{3}\right)$} \\
\hline After manual thrombectomy & & $70.0(51.8-86.7)$ \\
\hline After ELCA & & $87.7(66.9-106.3)$ \\
\hline After ballooning & & $121.2(93.3-152.9)$ \\
\hline \multicolumn{3}{|c|}{ Mean reference vessel area $\left(\mathrm{mm}^{3} / \mathrm{mm}\right)$} \\
\hline After manual thrombectomy & & $5.4 \pm 2.0$ \\
\hline After ELCA & & $6.0 \pm 2.0$ \\
\hline After ballooning & & $7.3 \pm 2.5$ \\
\hline \multicolumn{3}{|c|}{ Minimal lumen area $\left(\mathrm{mm}^{2}\right)$} \\
\hline After manual thrombectomy & & $1.4 \pm 0.7$ \\
\hline After ELCA & & $2.4 \pm 0.9$ \\
\hline After ballooning & & $5.6 \pm 2.0$ \\
\hline \multicolumn{3}{|c|}{ Culprit lesion morphology } \\
\hline Ruptured plaque & & $58(63.0 \%)$ \\
\hline Erosion & & $23(25.0 \%)$ \\
\hline Calcified nodule & & $7(7.6 \%)$ \\
\hline Unidentified & & $4(4.3 \%)$ \\
\hline \multicolumn{3}{|c|}{ Predominant type of thrombus } \\
\hline Red thrombus & & $60(65.2 \%)$ \\
\hline White thrombus & & $18(19.6 \%)$ \\
\hline Mixed thrombus & & $14(15.2 \%)$ \\
\hline
\end{tabular}

Data are presented as median (interquartile range), mean $\pm \mathrm{SD}$, or $n(\%)$. ELCA $=$ excimer laser coronary angioplasty.

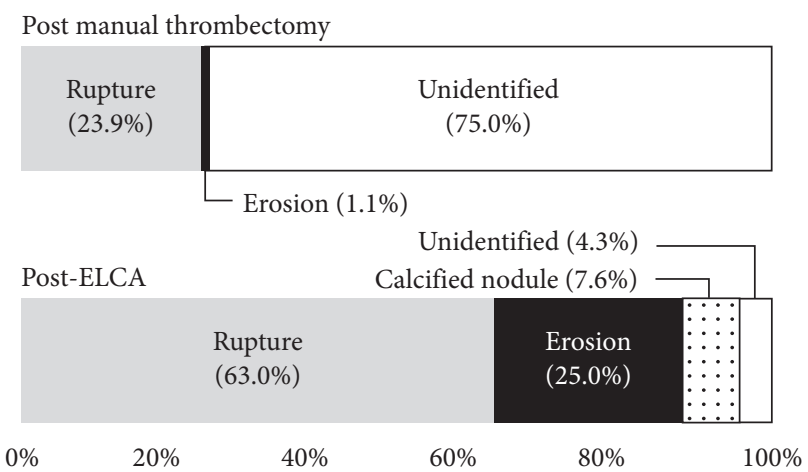

FIgURE 3: OCT identification of lesion morphology after MT and ELCA. Lesion morphology was not identifiable in the majority of cases even after MT because of the residual thrombus occupying the lumen. Vaporization of the thrombus by ELCA facilitated the identification of plaque morphology using OCT. OCT, optical coherence tomography; MT, manual aspiration thrombectomy; and ELCA, excimer laser coronary angioplasty.

incomplete stent coverage of the ruptured site is relatively common [24]. This insufficient lesion coverage appears to have a substantial clinical impact because the stent-uncovered ruptured plaque often exhibits chronic lumen reduction during the healing process [25]. In addition, although the rupture site appears to be adequately covered by the stent, chronic thrombus dissolution over time may result in late stent malapposition, which is significantly more frequent in ruptured plaque [26]. On the other hand, nonruptured plaque, such as lesions with plaque erosion, usually contains less thrombus burden and can be safely managed by oral dual antiplatelet therapy alone without intracoronary stenting [27]. Therefore, in vivo identification of culprit plaque morphology by reducing the intracoronary thrombus burden with ELCA may assist in developing an interventional approach for patients with STEMI.

4.1. Limitations. This was a single-center and nonrandomized study wherein ELCA was employed in all lesions. A further randomized study comparing PCI for STEMI with and without ELCA is required to identify the detailed benefits of ELCA. None of the patients were pretreated with a glycoprotein IIb/IIIa inhibitor, as this agent is not available in Japan. Potent antiplatelet or antithrombotic therapies may have influenced the efficacy of ELCA in vaporizing the intracoronary thrombus. The thrombus clot at the site of culprit lesion may, to some extent, embolize distally during insertion of the OCT or the laser catheter. Although the OCT catheter is smaller (3.0 Fr at the distal shaft) and the laser catheter is similar (5.3 Fr; $1.7 \mathrm{c})$ in size to the manual aspiration catheter $(5.1 \mathrm{Fr})$, the possibility that the reduced thrombus volume was simply because of insertion of any catheter into the coronary artery cannot be ruled out; this may have potentially altered the quantitative analysis of the thrombus volume. The number of patients included in the present study was relatively small, especially for assessing the distribution of culprit lesion morphology in STEMI. However, the sample size of 92 patients treated with ELCA and assessed by OCT to evaluate its efficacy is the largest population analyzed 

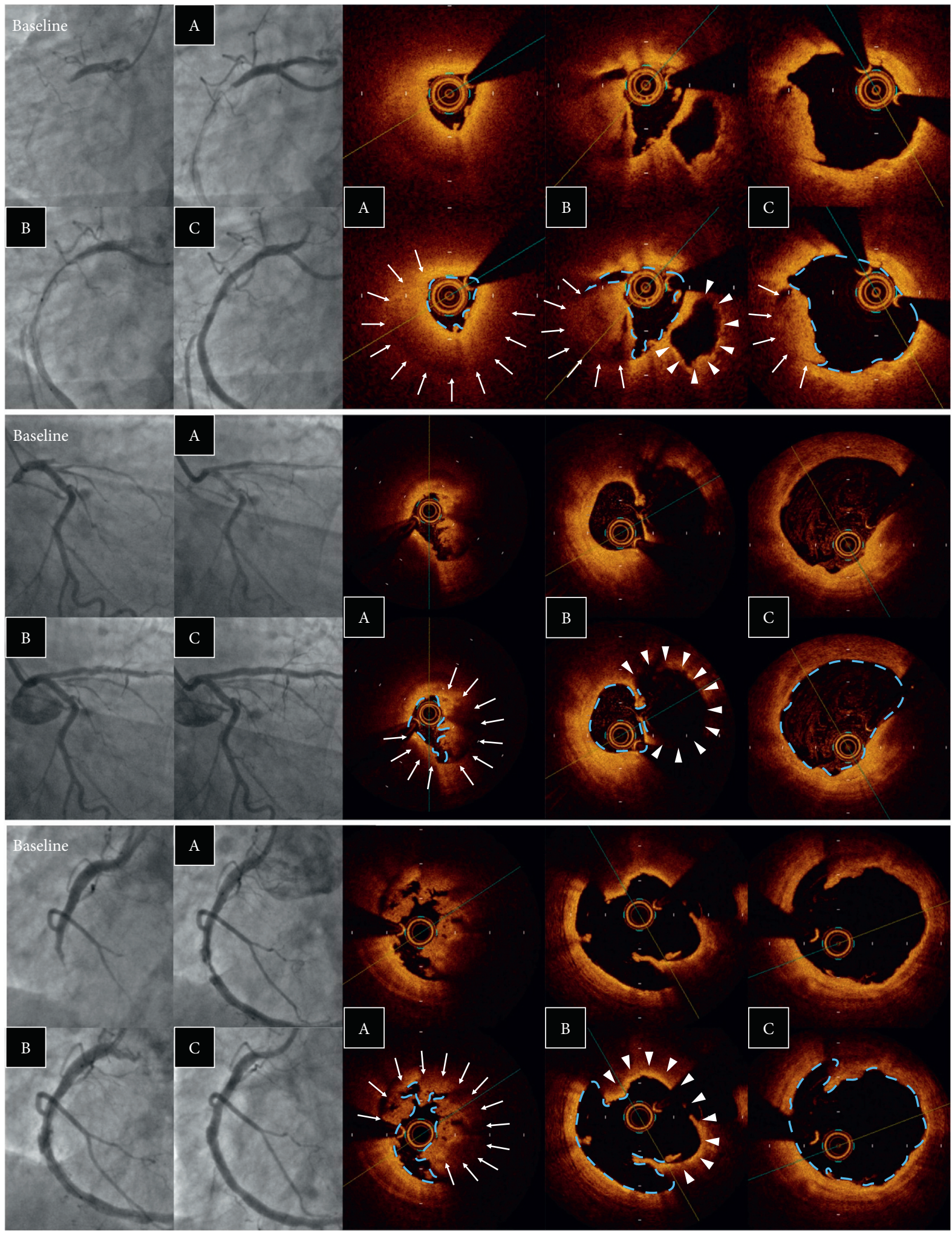

FIgURE 4: Examples of plaque rupture. Angiographic and OCT images after (A) MT, (B) ELCA, and (C) ballooning. Laser vaporization of the thrombus (arrow) enabled the identification of the ruptured cavity (arrow head). ELCA, excimer laser coronary angioplasty; MT, manual aspiration thrombectomy; and OCT, optical coherence tomography. 

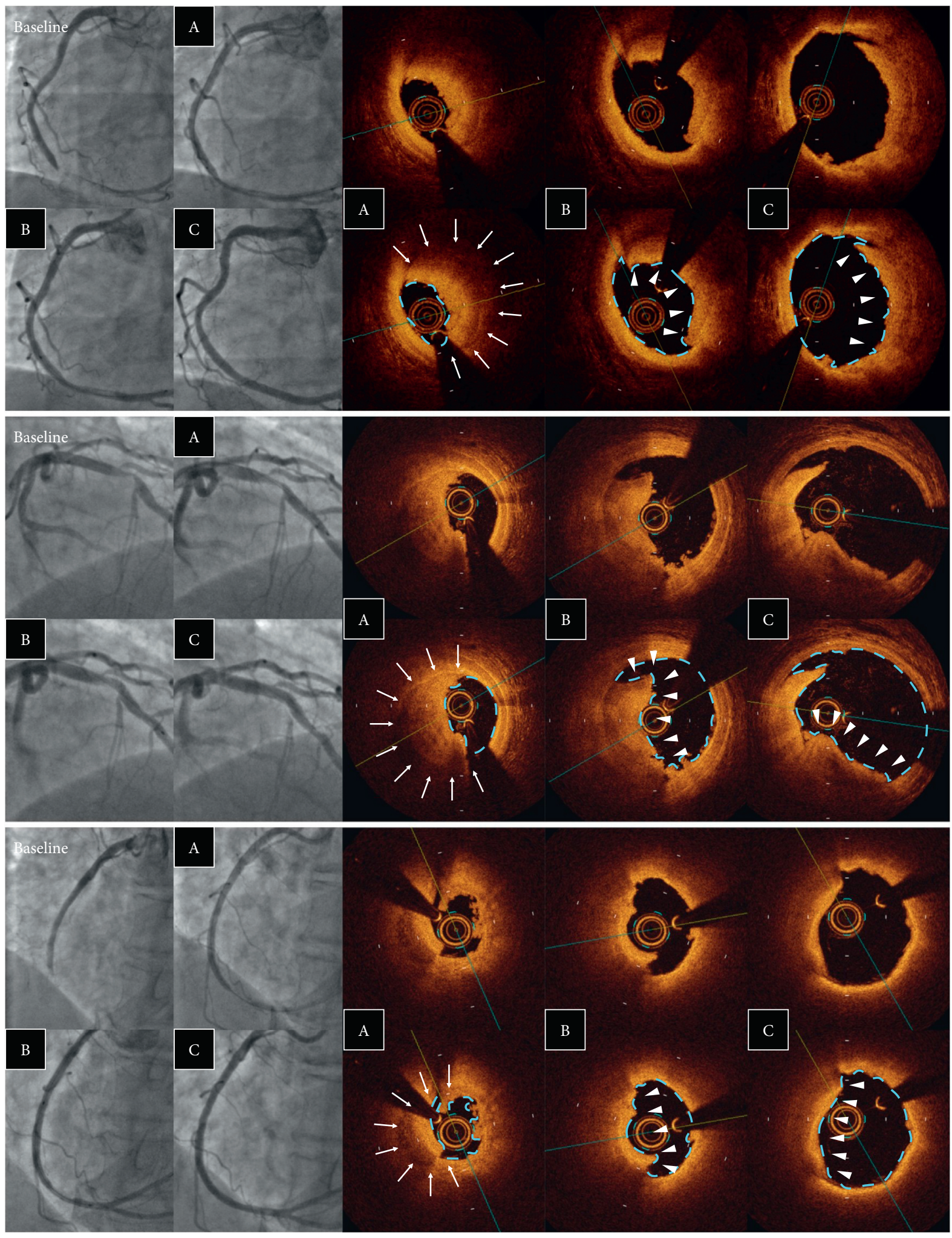

Figure 5: Examples of plaque erosion. Angiographic and OCT images after (A) MT, (B) ELCA, and (C) ballooning. Laser vaporization of the thrombus (arrow) clarified the identification of the attached thrombus overlying the eroded plaque (arrow head). ELCA, excimer laser coronary angioplasty; MT, manual aspiration thrombectomy; and OCT, optical coherence tomography. 

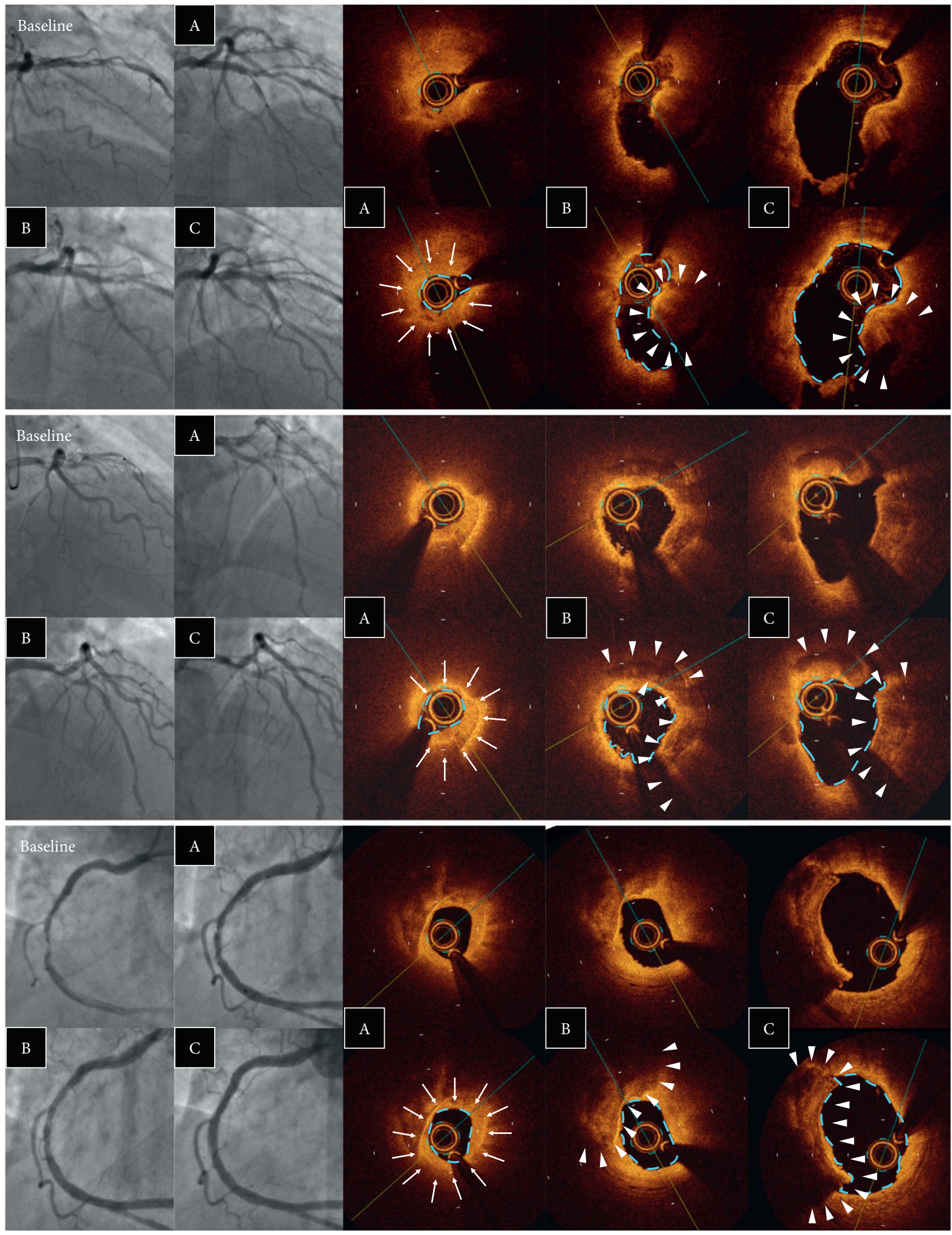

Figure 6: Examples of calcium nodules. Angiographic and OCT images after (A) MT, (B) ELCA, and (C) ballooning. Laser vaporization of the thrombus (arrow) clarified the identification of nodular protruding calcium (arrow head). ELCA, excimer laser coronary angioplasty; MT, manual aspiration thrombectomy; and OCT, optical coherence tomography. 
TABLE 4: Comparison between ruptured and nonruptured plaque.

\begin{tabular}{|c|c|c|c|}
\hline & Ruptured plaque $(n=58)$ & Nonruptured plaque $(n=31)$ & $p$ value \\
\hline $\begin{array}{l}\text { Predominant type of thrombus } \\
\text { Red thrombus } \\
\text { White thrombus } \\
\text { Mixed thrombus }\end{array}$ & $\begin{aligned} 44 & (75.9 \%) \\
5 & (8.6 \%) \\
9 & (15.5 \%)\end{aligned}$ & $\begin{array}{c}13(43.3 \%) \\
12(40.0 \%) \\
5(16.7 \%)\end{array}$ & 0.001 \\
\hline Thrombus length (mm) & $7.1 \pm 3.1$ & $6.9 \pm 4.2$ & 0.532 \\
\hline $\begin{array}{l}\text { Thrombus volume }\left(\mathrm{mm}^{3}\right) \\
\text { After manual thrombectomy } \\
\text { After ELCA } \\
\text { After ballooning }\end{array}$ & $\begin{array}{c}70.4(47.8-89.8) \\
46.2(30.4-62.8) \\
14.4(3.9-28.0) \\
\end{array}$ & $\begin{array}{c}49.5(33.9-70.0) \\
42.2(26.0-60.9) \\
12.7(4.5-31.7) \\
\end{array}$ & $\begin{array}{l}0.035 \\
0.495 \\
0.945 \\
\end{array}$ \\
\hline Reduction of thrombus volume by ELCA & $17.5(8.9-30.1)$ & $8.5(0.7-17.7)$ & 0.004 \\
\hline $\begin{array}{l}\text { Luminal volume }\left(\mathrm{mm}^{3}\right) \\
\text { After manual thrombectomy } \\
\text { After ELCA } \\
\text { After ballooning }\end{array}$ & $\begin{array}{c}69.5(52.3-90.4) \\
91.5(70.7-107.7) \\
128.2(102.8-160.9) \\
\end{array}$ & $\begin{aligned} & 70.6(51.4-80.4) \\
& 79.4(59.9-92.9) \\
& 106.4(79.8-123.9) \\
&\end{aligned}$ & $\begin{array}{l}0.676 \\
0.068 \\
0.014\end{array}$ \\
\hline $\begin{array}{l}\text { Minimal lumen area }\left(\mathrm{mm}^{2}\right) \\
\text { After manual thrombectomy } \\
\text { After ELCA } \\
\text { After ballooning }\end{array}$ & $\begin{array}{l}1.4 \pm 0.7 \\
2.5 \pm 1.0 \\
5.8 \pm 1.9\end{array}$ & $\begin{array}{l}1.4 \pm 0.7 \\
2.3 \pm 0.8 \\
5.0 \pm 1.9\end{array}$ & $\begin{array}{l}0.996 \\
0.588 \\
0.034\end{array}$ \\
\hline
\end{tabular}

Data are presented as $n(\%)$, median (interquartile range), or mean \pm SD. ELCA $=$ excimer laser coronary angioplasty.

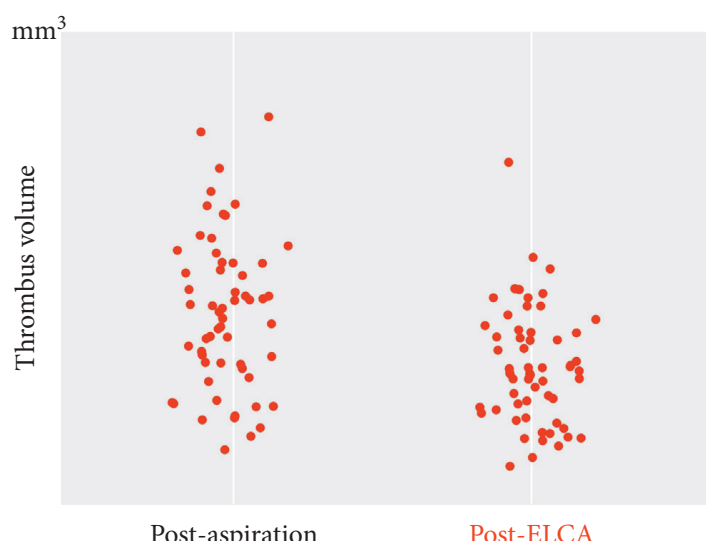

(a)

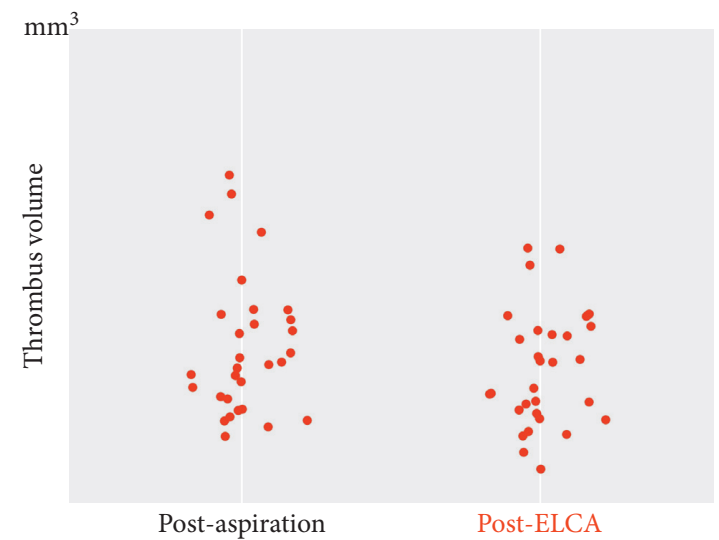

(b)

FiguRE 7: Scatter plots of thrombus volume after manual aspiration thrombectomy and laser vaporization. ELCA, excimer laser coronary angioplasty. (a) Ruptured plaque. (b) Nonruptured plaque.

with this technique thus far. Finally, the finding of more plaque rupture after ELCA may not only reflect greater clearance of thrombus but also be related to laser-induced intimal injury. Although previous studies demonstrated that atheroma mass is minimally ablated by ELCA and that ELCA-induced channels are relatively smooth without relevant histologic damage $[28,29]$, ELCA may have caused vascular injury and affected the identification of the lesion morphology.

\section{Conclusions}

In patients with STEMI, ELCA was feasible and effective in additionally reducing the intracoronary thrombus burden and creating a larger blood flow area even after MT. Moreover, the vaporization of the thrombus by ELCA also facilitated the identification of plaque morphology using OCT. Lesions with plaque rupture contained a larger thrombus burden that was frequently characterized by red thrombus and was more effectively reduced by ELCA compared to those with nonruptured plaque.

\section{Data Availability}

Data are available on reasonable request due to privacy/ ethical restrictions.

\section{Conflicts of Interest}

The authors declare that there are no conflicts of interest regarding the publication of this article. 


\section{Acknowledgments}

The authors would like to thank Enago (http://www.enago. jp) for the English language review.

\section{References}

[1] T. Higuma, T. Soeda, M. Yamada et al., "Does residual thrombus after aspiration thrombectomy affect the outcome of primary PCI in patients with ST-segment elevation myocardial infarction?" JACC: Cardiovascular Interventions, vol. 9, no. 19, pp. 2002-2011, 2016.

[2] S. S. Jolly, J. A. Cairns, S. Lavi et al., "Thrombus aspiration in patients with high thrombus burden in the total trial," Journal of the American College of Cardiology, vol. 72, no. 14, pp. 1589-1596, 2018.

[3] G. Sardella, M. Mancone, C. Bucciarelli-Ducci et al., "Thrombus aspiration during primary percutaneous coronary intervention improves myocardial reperfusion and reduces infarct size," Journal of the American College of Cardiology, vol. 53, no. 4, pp. 309-315, 2009.

[4] T. Svilaas, P. J. Vlaar, I. C. van der Horst et al., "Thrombus aspiration during primary percutaneous coronary intervention," New England Journal of Medicine, vol. 358, no. 6, pp. 557-567, 2008.

[5] P. J. Vlaar, T. Svilaas, I. C. van der Horst et al., "Cardiac death and reinfarction after 1 year in the thrombus aspiration during percutaneous coronary intervention in acute myocardial infarction Study (TAPAS): a 1-year follow-up study," The Lancet, vol. 371, no. 9628, pp. 1915-1920, 2008.

[6] B. Lagerqvist, O. Fröbert, G. K. Olivecrona et al., "Outcomes 1 year after thrombus aspiration for myocardial infarction," New England Journal of Medicine, vol. 371, no. 12, pp. 1111-1120, 2014.

[7] Y. Onuma, L. Thuesen, R.-J. van Geuns et al., "Randomized study to assess the effect of thrombus aspiration on flow area in patients with ST-elevation myocardial infarction: an optical frequency domain imaging study-TROFI trial," European Heart Journal, vol. 34, no. 14, pp. 1050-1060, 2013.

[8] R. Bhindi, O. A. Kajander, S. S. Jolly et al., "Culprit lesion thrombus burden after manual thrombectomy or percutaneous coronary intervention-alone in ST-segment elevation myocardial infarction: the optical coherence tomography substudy of the total (Thrombectomy versus PCI alone) trial," European Heart Journal, vol. 36, no. 29, pp. 1892-1900, 2015.

[9] O. Topaz, D. Ebersole, T. Das et al., "Excimer laser angioplasty in acute myocardial infarction (the carmel multicenter trial)," The American Journal of Cardiology, vol. 93, no. 6, pp. 694701, 2004.

[10] M. Dörr, D. Vogelgesang, A. Hummel et al., "Excimer laser thrombus elimination for prevention of distal embolization and no-reflow in patients with acute ST elevation myocardial infarction," International Journal of Cardiology, vol. 116, no. 1, pp. 20-26, 2007.

[11] V. Ambrosini, A. Cioppa, L. Salemme et al., "Excimer laser in acute myocardial infarction: single centre experience on 66 patients," International Journal of Cardiology, vol. 127, no. 1, pp. 98-102, 2008.

[12] O. Topaz, D. Ebersole, and J. B. Dahm, "Excimer laser in myocardial infarction: a comparison between STEMI patients with established $Q$-wave versus patients with non-STEMI (non-Q)," Lasers in Medical Science, vol. 23, no. 1, pp. 1-10, 2008.
[13] N. Amabile, S. Hammas, S. Fradi et al., "Intra-coronary thrombus evolution during acute coronary syndrome: regression assessment by serial optical coherence tomography analyses," European Heart Journal-Cardiovascular Imaging, vol. 16, no. 4, pp. 433-440, 2015.

[14] F. Prati, E. Regar, G. S. Mintz et al., "Expert review document on methodology, terminology, and clinical applications of optical coherence tomography: physical principles, methodology of image acquisition, and clinical application for assessment of coronary arteries and atherosclerosis," European Heart Journal, vol. 31, no. 4, pp. 401-415, 2010.

[15] O. A. Kajander, L. S. Koistinen, M. Eskola et al., "Feasibility and repeatability of optical coherence tomography measurements of pre-stent thrombus burden in patients with STEMI treated with primary PCI," European Heart JournalCardiovascular Imaging, vol. 16, no. 1, pp. 96-107, 2015.

[16] K. D. Taylor and C. Reiser, "From laser physics to clinical utilization: design and ablative properties of cardiovascular laser catheters," Lasers in Cardiovascular Interventions, Springer, London, UK, pp. 1-14, 2015.

[17] W. S. Grundfest, F. Litvack, J. S. Forrester et al., "Laser ablation of human atherosclerotic plaque without adjacent tissue injury," Journal of the American College of Cardiology, vol. 5, no. 4, pp. 929-933, 1985.

[18] E. Ilkay, I. Karaca, M. Yavuzkir, M. Akbulut, and M. Pekdemir, "The effect of interventional treatment in acute myocardial infarction on ST resolution: a comparison of coronary angioplasty with excimer laser angioplasty," Angiology, vol. 56, no. 4, pp. 377-384, 2005.

[19] D. Shishikura, S. Otsuji, S. Takiuchi et al., "Vaporizing thrombus with excimer laser before coronary stenting improves myocardial reperfusion in acute coronary syndrome," Circulation Journal, vol. 77, no. 6, pp. 1445-1452, 2013.

[20] S. Hammas, A. Veugeois, C. Caussin, and N. Amabile, "Analysis of residual thrombotic burden after thrombus aspiration in acute ST elevation myocardial infarction: an optical coherence tomographic evaluation," International Journal of Cardiology, vol. 181, pp. 351-352, 2015.

[21] E. Falk, M. Nakano, J. F. Bentzon, A. V. Finn, and R. Virmani, "Update on acute coronary syndromes: the pathologists' view," European Heart Journal, vol. 34, no. 10, pp. 719-728, 2013.

[22] F. Saia, K. Komukai, D. Capodanno et al., "Eroded versus ruptured plaques at the culprit site of STEMI," JACC: Cardiovascular Imaging, vol. 8, no. 5, pp. 566-575, 2015.

[23] J. Legutko, J. Jakala, G. S. Mintz et al., "Virtual histologyintravascular ultrasound assessment of lesion coverage after angiographically-guided stent implantation in patients with ST elevation myocardial infarction undergoing primary percutaneous coronary intervention," The American Journal of Cardiology, vol. 109, no. 10, pp. 1405-1410, 2012.

[24] M. Hougaard, H. S. Hansen, P. Thayssen, L. Antonsen, and L. O. Jensen, "Uncovered culprit plaque ruptures in patients with ST-segment elevation myocardial infarction assessed by optical coherence tomography and intravascular ultrasound with iMap," JACC: Cardiovascular Imaging, vol. 11, no. 6, pp. 859-867, 2018.

[25] P. A. Calvert, A. J. Brown, S. P. Hoole, D. R. Obaid, N. E. J. West, and M. R. Bennett, "Geographical miss is associated with vulnerable plaque and increased major adverse cardiovascular events in patients with myocardial infarction," Catheterization and Cardiovascular Interventions, vol. 88, no. 3, pp. 340-347, 2016. 
[26] L. Wang, G. Parodi, A. Maehara et al., "Variable underlying morphology of culprit plaques associated with ST-elevation myocardial infarction: an optical coherence tomography analysis from the SMART trial," European Heart JournalCardiovascular Imaging, vol. 16, no. 12, pp. 1381-1389, 2015.

[27] L. Xing, E. Yamamoto, T. Sugiyama et al., "Erosion study (effective anti-thrombotic therapy without stenting: intravascular optical coherence tomography-based management in plaque erosion): a 1-year follow-up report," Circulation Cardiovascular Interventions, vol. 10, no. 12, Article ID e005860, 2017.

[28] T. A. Sanborn, D. Alexopoulos, J. D. Marmur et al., "Coronary excimer laser angioplasty: reduced complications and indium-111 platelet accumulation compared with thermal laser angioplasty," Journal of the American College of Cardiology, vol. 16, no. 2, pp. 502-506, 1990.

[29] J. Honye, D. J. Mahon, S. Nakamura et al., "Intravascular ultrasound imaging after excimer laser angioplasty," Catheterization and Cardiovascular Diagnosis, vol. 32, no. 3, pp. 213-222, 1994. 